\title{
Avaliação da resistividade elétrica do concreto utilizando o método direto
}

\author{
LIDIANNE DO NASCIMENTO FARIAS - Mestre Em Engenharia CIIIL E Ambiental \\ PAULO ROBERTO LOPES LIMA - PROFESSOR DOUTOR \\ CINTIA MARIA ARIANI FONTES - ProfESSORA DoUTORA \\ ANFRANSERAI MORAIS DIAS - ProfESSOR Doutor \\ Universidade Estadual de Feira de Santana (UEFS)
}

\section{RESUMO}

O PRESENTE ESTUDO TEVE COMO OBJETIVO AVALIAR A RESISTIVIDADE ELÉTRICA DE CONCRETOS COM DIFERENTES TEORES DE AGREGADO GRAÚDO (EM 40\%, 43\% E 46\%), UTILIZANDO O MÉTODO DIRETO PRESCRITO PELA NORMA ESPANHOLA UNE 83988-1 E COM O AUXÍLIO DE UM APARATO DESENVOLVIDO EM LABORATÓRIO. AlÉM DA VARIAÇÃ̃ DA COMPOSIÇÃO DO CONCRETO, DOIS PARÂMETROS ASSOCIADOS AO ENSAIO FORAM INVESTIGADOS: TEOR DE UMIDADE E DIMENSÃO DA AMOSTRA. OS RESULTADOS INDICAM UM AUMENTO DA RESISTIVIDADE ELÉTRICA VOLUMÉTRICA COM O AUMENTO DO TEOR DE AGREGADO E

AUMENTO DA UMIDADE DO CONCRETO. A VARIAÇÃO DA UMIDADE DAS AMOSTRAS, DE 44\% A 100\%, INDICOU UMA REDUÇÃO DA RESISTIVIDADE ELÉTRICA

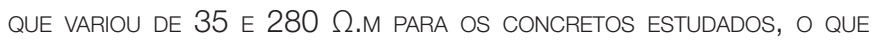
IMPLICA AUMENTO DO RISCO DE CORROSÃO DE ARMADURAS QUE PASSOU DE MODERADO PARA MUITO ALTO. A AVALIAÇÃO DA DIMENSÃO DA AMOSTRA INDICA UM AUMENTO DA RESISTIVIDADE ELÉTRICA VOLUMÉTRICA À MEDIDA QUE HÁ UMA REDUÇÃO DA ALTURA DA AMOSTRA, SENDO PROPOSTO UM FATOR DE CORREÇÃO PARA COMPARAR ENSAIOS REALIZADOS COM AMOSTRAS DIFERENTES.

Palavras-chave: durabilidade, resistividade elétrica volumétrica, dimensão da amostra, risco de corrosão, umidade do concreto.

\section{INTRODUÇ̃̃O}

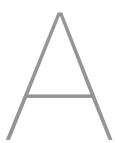

avaliação periódica da durabilidade do concreto

é uma das etapas fundamentais para o monitoramento da qualidade das estruturas de concreto armado e definição de ações de manutenção que garantam que a vida útil de projeto seja atingida. Dentre os diversos ensaios disponíveis, a medida da resistividade elétrica se apresenta como um método acessível e confiável para avaliação da possiblidade de risco de corrosão das armaduras, devido à carbonatação do concreto ou à penetração de cloretos. A medida de resistividade elétrica representa a dificuldade com que a corrente elétrica flui pelo interior do concreto e, nesse sentido, pode ser utilizada como uma medida indireta da capacidade do concreto em resistir à penetração de agentes agressivos que possibilitam a corrosão das armaduras. Com isso, a resistividade elétrica do concreto pode ser correlacionada com o modelo de vida útil das estruturas, pois, apesar de não mensurar a corrosão do aço, indica a sua possibilidade de ocorrência.

A medida de resistividade é afetada diretamente por parâmetros associados à composição do concreto, como relação água/cimento, teor e tipo de cimento e teor de agregado, e por parâmetros associados ao ensaio, como teor de umidade, dimensão da amostra e estado de fissuração da amostra, no caso de testemunhos de concreto.
Como a corrente elétrica é transportada por íons que fluem através da umidade presente nesses poros, o teor de umidade mais alto facilita a mobilidade iônica e reduz a resistividade elétrica.

O método mais usado no Brasil para a determinação da resistividade elétrica é o ensaio de quatro pontos, que tem a facilidade de ser utilizado em campo, mas que tem a limitação de medir a resistividade superficial do concreto, e não do seu interior. Devido a isso, várias medidas têm que ser feitas ao longo da superfície da amostra para aumentar a representatividade do ensaio e reduzir a variabilidade dos resultados. Como utiliza quatro pinos para gerar a diferença de potencial e monitorar a tensão, que são espaçados usualmente por $5 \mathrm{~cm}$, 
Tabela 1 - Concretos utilizados

\begin{tabular}{|ccccccc|} 
Mistura & $\begin{array}{c}\text { Cimento } \\
\left(\mathrm{Kg} / \mathrm{m}^{3}\right)\end{array}$ & $\begin{array}{c}\text { Areia } \\
\left(\mathrm{Kg} / \mathrm{m}^{3}\right)\end{array}$ & $\begin{array}{c}\text { Brita } \\
\left(\mathrm{Kg} / \mathrm{m}^{3}\right)\end{array}$ & $\begin{array}{c}\text { Água } \\
\left(\mathrm{Kg} / \mathrm{m}^{3}\right)\end{array}$ & $\begin{array}{c}\text { Relaç̃̃̃o } \\
\text { a/c }\end{array}$ & $\begin{array}{c}\mathrm{f}_{\mathrm{c}}-\mathrm{C} . \mathrm{V} . \\
(\mathrm{M} \mathrm{Pa})(\%)\end{array}$ \\
\hline C40 & 378 & 824,0 & 929,9 & 207,9 & 0,55 & $23,9-3,5$ \\
\hline C43 & 364 & 793,5 & 1033,8 & 200,2 & 0,55 & $26,7-1,0$ \\
\hline C46 & 351 & 765,2 & 1137,2 & 193,1 & 0,55 & $24,7-0,3$ \\
\hline
\end{tabular}

o método exige que as amostras tenham maior comprimento, o que pode inviabilizar seu uso em testemunhos de concreto extraídos de estruturas esbeltas. Uma alternativa é o método direto, que é aplicado em dois lados opostos da amostra, e consegue determinar a resistividade elétrica volumétrica do concreto, o que representa melhor suas características. Além da facilidade de aplicação em laboratório, o método pode ser aplicado em testemunhos de dimensões menores que os utilizados no método superficial, o que amplia sua possibilidade de uso. No Brasil, a determinação da resistividade elétrica pelo método direto é apresentada pela ABNT NBR 9204 que, devido ao uso de eletrodos constituídos de mercúrio metálico líquido, é limitado devido a problemas de saúde pública e ambientais. Neste trabalho, a resistividade elétrica de concretos com diferentes composições foi determinada pelo método direto proposto pela norma espanhola UNE 83988-1 (AENOR, 2008). Em adição, foram investigados o efeito do teor de umidade e a dimensão da amostra sobre os valores de resistividade elétrica.

\section{RELAÇÃO ENTRE RESISTIVIDADE ELÉTRICA E DURABILIDADE DO CONCRETO}

A resistividade elétrica $\rho(\Omega . m)$ indica a restrição à passagem de carga elétrica pelo interior do material e, dessa forma, pode ser relacionada com a permeabilidade e difusão, que são os principais mecanismos de transporte dos agentes agressivos. No estado saturado, o concreto é um elemento semicondutor e, no estado seco, pode ser considerado quase isolante.

Desse modo, a medida de resistividade elétrica do concreto pode ser utilizada na determinação indireta da conectividade dos poros, pois define a capacidade do concreto em permitir a transferência de íons através da sua microestrutura, permitindo avaliar a possível movimentação de cloretos através dos poros do concreto endurecido (MENDES et al, 2018) e prever a possibilidade de corrosão das armaduras (MEDEIROS-JUNIOR e LIMA, 2016).

A resistividade elétrica do concreto está ligada a cinética de corrosão das armaduras e a sua determinação pode atuar como forma de avaliação da pro-

teção da camada de cobrimento do concreto no elemento de concreto armado.

\section{PROGRAMA EXPERIMENTAL}

Foram produzidos três diferentes tipos de concreto, com variação no teor de agregado graúdo (40\%, 43\% e 46\%), como mostra a Tabela 1. Utilizou-se cimento Portland CPII-F32, areia quartzosa com módulo de finura de 2,52 e brita granítica com dimensão máxima característica de $19 \mathrm{~mm}$.

A resistência à compressão dos concretos $\left(f_{c}\right)$ foi determinada em três corpos de prova cilíndricos $(100 \mathrm{~mm}$ de diâmetro x 200 mm de altura), curados em água até os 28 dias de idade, conforme norma NBR 5739, e está apresentada na Tabela 1.

A resistividade elétrica foi determinada de acordo com a norma UNE 83988-1 (AENOR, 2008), em um aparato montado em laboratório, como mostra a Figura 1.

Para montagem do aparato, foi utilizada uma fonte com uma frequência estabelecida entre 82 - $85 \mathrm{~Hz}$. Utilizou-se também dois multímetros digitais, um para medição da corrente alternada e outro para mediação da tensão. A tensão obtida variou entre 7,30 7,40V. Os eletrodos foram compostos

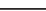

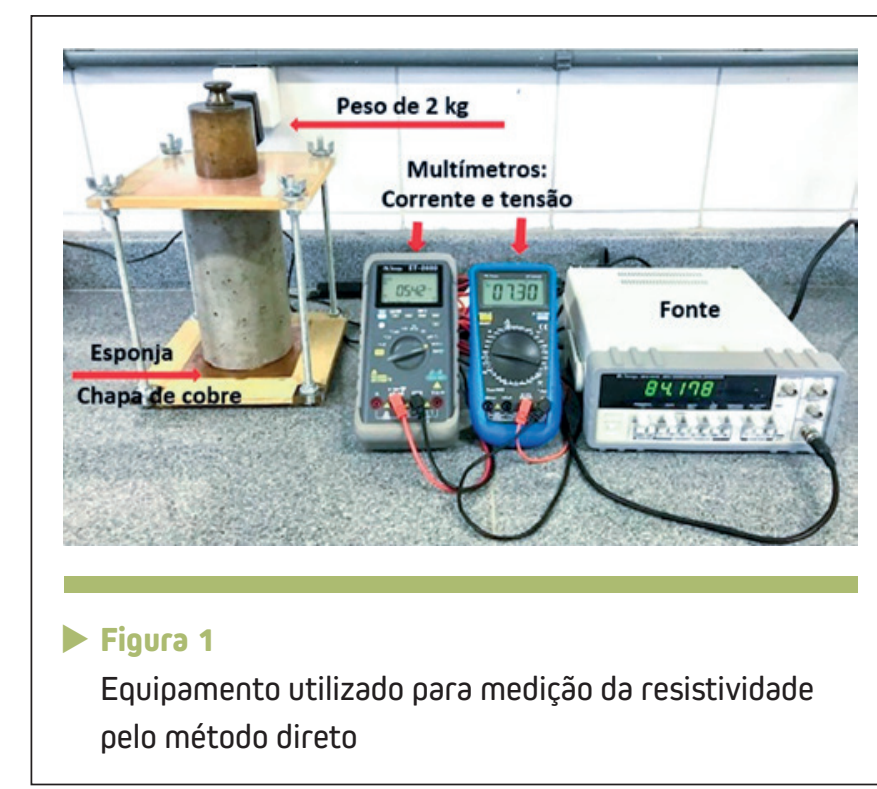


por duas chapas de cobre e por duas esponjas úmidas, para manter uma melhor conexão, que foram acoplados paralelamente ao corpo de prova. Para melhorar o contato da chapa de cobre com a superfície do concreto e facilitar a passagem da corrente, foi colocado um peso de balança de $2 \mathrm{~kg}$ sobre a amostra.

O aparato desenvolvido foi calibrado com uma sonda de Wenner, com espaçamento entre sondas de $50 \mathrm{~mm}$, em que diversos corpos de prova foram testados simultaneamente pelos dois métodos, sendo encontrado a relação:

$\rho_{\text {superficial }}=1,9 \rho_{\text {volunétrico }}$

Em que a $\rho_{\text {superficial }}$ é a resistividade elétrica medida pela sonda de Wenner e $\rho_{\text {volunétrico }}$ é a resistividade medida pelo método direto. O fator de determinação $R^{2}$ foi igual a 0,96 .

Azarsa e Gupta (2017) mostraram que os valores obtidos entre os dois tipos de ensaio são proporcionais, sendo que o valor de resistividade volumétrica corresponde à metade do valor obtido pelo método de Wenner. No entanto, alguns fatores associados aos ensaios

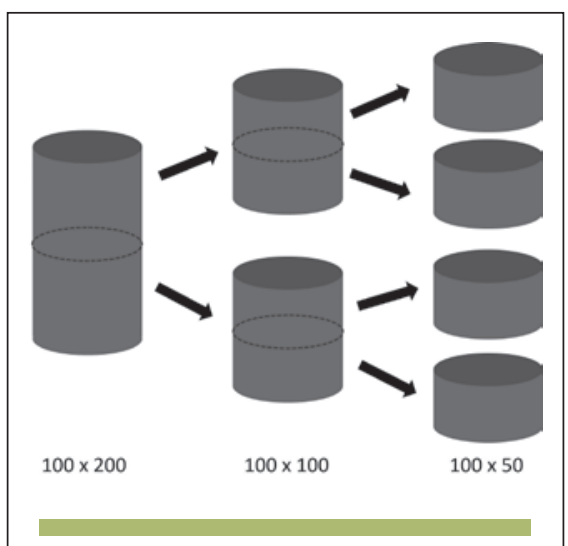

Figura 2

Redução da dimensão (em mm) do corpo de prova para ensaio de resistividade elétrica podem modificar essa relação, como o espaçamento das sondas, a dimensão da amostra e mesmo as tensões adotadas no método direto. Os valores obtidos por cada ensaio não são iguais devido à forma como a corrente elétrica se distribui na amostra, no entanto, a adoção de padrões para cada método de ensaio, através de normas técnicas específicas, permitirá estabelecer um valor de correlação que possa ser empregado de forma segura e confiável.

A resistividade elétrica inicialmente foi medida aos 28 dias de idade em concretos com diferentes teores de umidades. As amostras foram colocadas em estufa até a constância de massa, colocadas ao ar para resfriamento e depois imersas em água até a saturação. Dessa forma, a resistividade elétrica foi medida, a partir do estado seco, em intervalos de 60 minutos durante as primeiras 6 horas em água, e depois com 24 horas, 48 horas e 72 horas de imersão.

A análise da dimensão da amostra sobre a resistividade elétrica foi realizada em corpos de prova cilíndricos de $100 \mathrm{~mm} \times 200 \mathrm{~mm}$, que foram posteriormente cortados com altura de 100 $\mathrm{mm}$, para uma segunda medida de resistividade, e com altura $50 \mathrm{~mm}$, para uma terceira medição, como mostra a Figura 2.

\section{RESULTADOS E DISCUSSÕES}

O efeito do teor de agregado graúdo e idade do concreto sobre a resistividade elétrica volumétrica do concreto, obtida com amostras 100\% saturadas, é apresentado na Figura 3.

Verifica-se um aumento da resistividade de cerca de $11 \%$ quando o teor de agregado mudou de $40 \%$ para $46 \%$. Shi (2004) confirmou o aumento da resistividade elétrica com o aumento do volume de agregado na mistura devido ao menor teor de pasta e, consequentemente, menos "espaço" para a mobilidade iônica. Um modelo apresentado por Hou et al. (2017) indica que a condução elétrica no concreto é feita por três caminhos possíveis como mostra a Figura 4: i) através do agregado em série (caminho A); ii) através da pasta de cimento, contornando agregados e vazios (caminhos $B$ e D) e iii) através dos grãos dos agregados com contato entre si (caminho C). O agregado apresenta menor capacidade de condução que a pasta de cimento, que possul maior umidade e, portanto, o aumento da quantidade de grãos aumenta a resistividade elétrica do concreto.

As alterações na pasta de cimento, por outro lado, são mais efetivas na modificação da resistividade elétrica (MEDEIROS-JUNIOR e LIMA, 2016). O aumento da idade do concreto, que indica um avanço do processo de hidratação da pasta de cimento, aumenta a resistividade elétrica como mostra a Figura 3. Outro fator relevante associado à fase pasta é a presença de poros que dificultam a condução elétrica dentro do

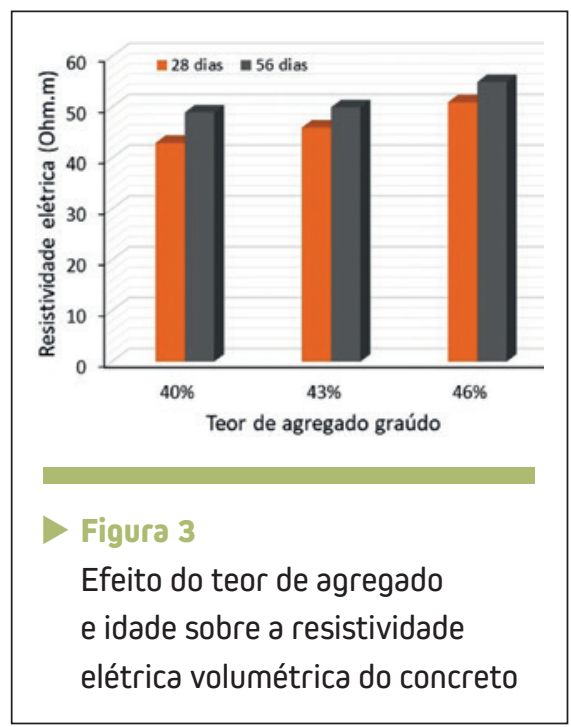




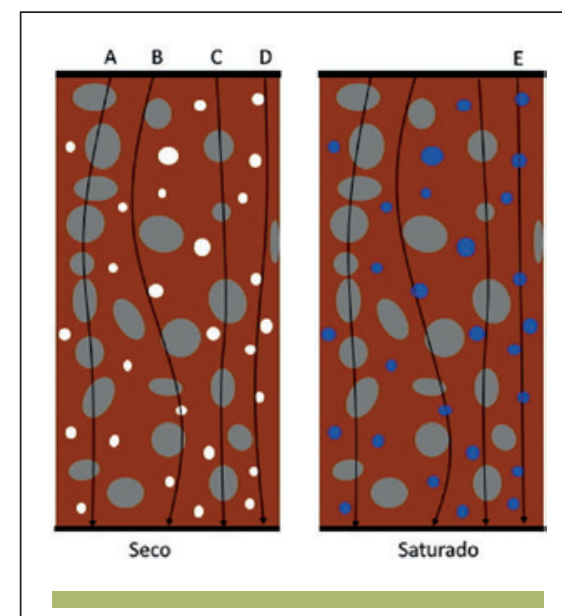

Figura 4

Modelo de condução elétrica em concretos

Fonte: adaptado de HOU et al., 2017

concreto, como mostra a linha D na Figura 4. Por outro lado, o aumento do teor de umidade do concreto faz com que os poros saturados se tornem condutores de corrente elétrica (ver linha E na Figura 4) e reduzam a resistividade elétrica.

O efeito do teor de umidade do concreto sobre a resistividade elétrica volumétrica é mostrado na Figura 5, onde verifica-se uma correlação inversamente proporcional entre a resistividade elétrica e o teor de umidade, independentemente do tipo da composição do concreto. Para teores de umidade menores que $40 \%$, a resistividade elétrica é muito alta e não foi medida pelo equipamento, enquanto para teores de umidade maiores que $40 \%$, a resistivida-

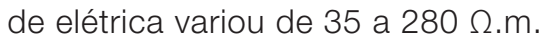

A resistividade elétrica pode ser utilizada para avaliar a qualidade do concreto e indicar o possível risco de corrosão da armadura, como mostra a Tabela 2. Isso permite realizar ações para proteção da armadura, no caso de estruturas em uso, ou para modificação da dosagem do concreto, antes da sua aplicação em estruturas. Utilizando a Tabela 2 e os valores obtidos de resistividade elétrica volumétrica, é possível também correlacionar o risco de corrosão ao teor de umidade do concreto, como mostra a Figura 5.

A Figura 6, que considera os valores de umidade associados aos riscos de corrosão mostrados na Figura 5, indica que, para um teor de umidade menor que 60\%, todos os concretos apresentaram risco de corrosão moderado. À medida que há incremento no teor de umidade, é verificado um aumento da possibilidade de corrosão, sendo que um risco muito alto já é verificado para

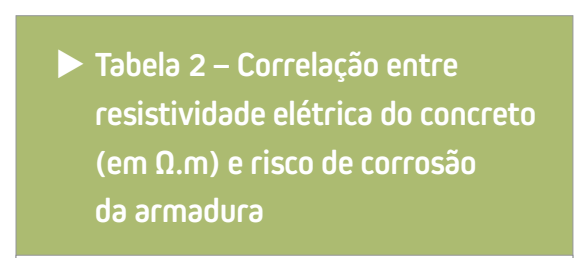

\begin{tabular}{|c|c|c|}
\hline $\begin{array}{l}\text { Resistividade } \\
\text { elétrica } \\
\text { superficial } \\
\text { (Balestra } \\
\text { et al., 2019) }\end{array}$ & $\begin{array}{l}\text { Resistividade } \\
\text { elétrica } \\
\text { volumétrica } \\
\text { (usando } \\
\text { Eq. 1) }\end{array}$ & $\begin{array}{l}\text { Risco de } \\
\text { corrosão }\end{array}$ \\
\hline$>800$ & $>420$ & Desprezível \\
\hline $550-800$ & $290-420$ & Baixo \\
\hline $200-550$ & $105-290$ & Moderado \\
\hline $150-200$ & $80-105$ & Alto \\
\hline$<150$ & $<80$ & Muito alto \\
\hline
\end{tabular}

teor de umidade maior que 68\%, a depender do teor de agregado. Nessa faixa de umidade, a resistividade elétrica volumétrica parece ser menos afetada pelo incremento do teor de umidade, indicando que os poros já saturados são suficientes para a condução elétrica entre os extremos da amostra, como mostra a curva E da Figura 4.

A possibilidade de aumento do risco de corrosão com o teor de umidade do concreto está de acordo com as prescrições da norma brasileira de estruturas de concreto armado ABNT NBR 6118, que indica que, quanto maior a

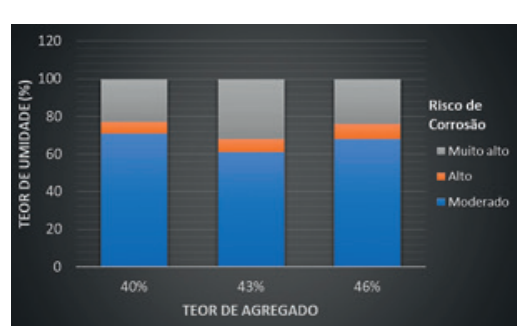

Figura 6

Influência da umidade sobre 0 risco de corrosão de armadura em diferentes concretos

\section{Variação da resistividade elétrica volumétrica com o aumento da umidade do concreto}


umidade do ambiente, maior a espessura de cobrimento necessária para proteger a armadura dentro do concreto, já que o teor de saturação dos poros do concreto determina o mecanismo da corrosão.

A influência da dimensão do corpo de prova sobre a resistividade elétrica do concreto é mostrada na Figura 7, considerando amostras com 100\% de umidade. Esta avaliação tem o objetivo de verificar a aplicação do método para testemunhos com menores dimensões, que possam ser extraídas de estruturas de concreto em uso, sem que causem maiores danos.

Com relação ao corpo de prova padrão, de dimensão 100 mm x 200 mm, verifica-se um aumento de aproximadamente 30\% em relação ao ganho de resistividade para amostras cilíndricas com menor altura. $\mathrm{O}$ efeito da dimensão da amostra sobre a resistividade elétrica do concreto já foi verificado por vários pesquisadores, sendo necessário a adoção de fatores de forma que possam associar a resistividade obtida em amostras com variadas di-

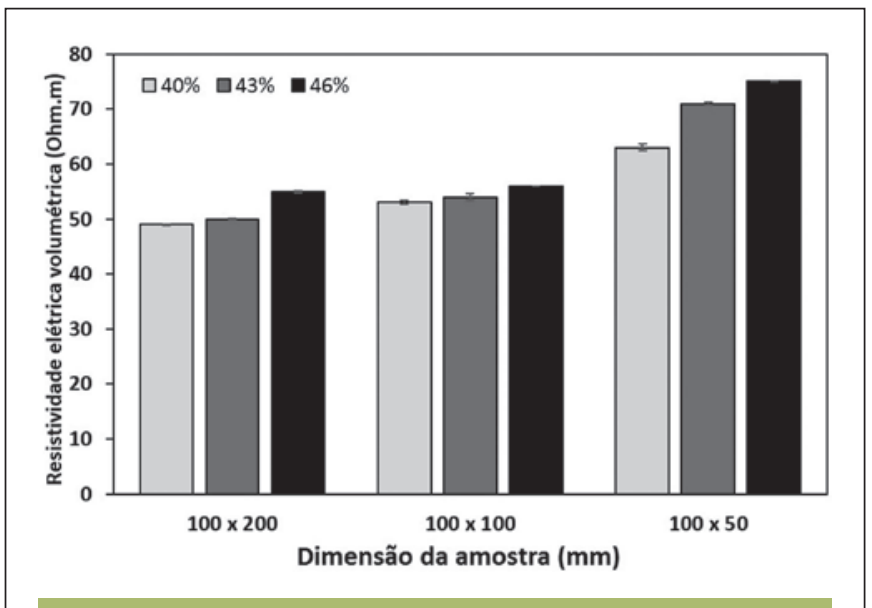

Figura 7

Efeito da dimensão da amostra na resistividade elétrica pelo método direto mensões com aquelas utilizadas em ensaio padrão.

$\rho_{e}=\frac{\rho_{e, c p}}{F C}$

Em que $\rho_{e}$ é a resistividade elétrica obtida de forma direta em amostras cilíndricas de dimensão 100 × 200 mm, conforme a UNE 83988-1 (AENOR, 2008), $\rho_{e, c p}$ é a resistividade elétrica obtida em amostras cilíndricas com outras dimensões e FC é o fator de correção mostrado na Tabela 3. No entanto, novos estudos ainda são necessários para validação dos fatores propostos.

\section{CONSIDERAÇÕES FINAIS}

A resistividade elétrica volumétrica pode ser considerada um padrão útil de avaliação da durabilidade de estruturas, principalmente, quando diz respeito a previsão da corrosão das armaduras. Aplicada em laboratório, essa técnica pode ser utilizada para avaliação da qualidade do concreto quanto ao risco de corrosão das armaduras, sendo importante na dosagem de novos concretos.

Os resultados apresentados neste trabalho demonstraram que a resistividade elétrica é sensível ao teor de agregado e a idade do concreto, demonstrando que a modificação na composição do concreto permite a dosagem de
> Tabela 3 - Fator de correção da resistividade elétrica, com relação a amostra cilíndrica de 1 $00 \mathrm{~mm}$ x $200 \mathrm{~mm}$

\begin{tabular}{|c|c|c|}
\hline \multicolumn{2}{|c|}{ Dimensão da amostra } & Fator de \\
\hline $\begin{array}{c}\text { Altura } \\
(\mathrm{mm})\end{array}$ & $\begin{array}{c}\text { Diâmetro } \\
(\mathrm{mm})\end{array}$ & \begin{tabular}{c} 
correção FC \\
\hline 50
\end{tabular} \\
\hline 100 & 100 & 1,36 \\
\hline
\end{tabular}

um material que propicie maior resistência à corrosão das armaduras.

Apesar de ser determinada em amostras cilíndricas de dimensão padrão 100 x 200 mm, os resultados obtidos indicam que é possível avaliar a resistividade elétrica em amostras menores e, através de um fator de correção apropriado, correlacionar os resultados. Essa constatação permite ampliar a utilização do método para o monitoramento de estruturas em uso que apresentem extração de testemunhos de pequena dimensão, como lajes.

Por atuar em função do fluxo de corrente elétrica através da água contida nos poros dos concretos, a resistividade elétrica é muito sensível ao seu teor de umidade. Neste estudo, as amostras analisadas obtiveram variações da umidade de 44\% a 100\%, indicando uma redução da resistividade e aumento do risco de corrosão de armaduras embutidas no concreto. Para os concretos estudados, verifica-se que o aumento do teor de umidade modifica a classificação do risco de corrosão, que passa de moderado para muito alto.

Verifica-se, portanto, que o método direto para determinação da resistividade elétrica do concreto se 
